Research Article

\title{
Adaptive Clustering-Based Marine Radar Sea Clutter Normalization
}

\author{
Yong Xu $\mathbb{D}^{1},{ }^{1}$ Tao Jia $\mathbb{D}^{1},{ }^{1}$ Dong Cao $\mathbb{D}^{2},{ }^{2}$ Pengyu Guo $\mathbb{D}^{3},{ }^{3}$ Yue Ma $\mathbb{D}^{1},{ }^{1}$ and Hongtao Yan $\mathbb{D}^{1}$ \\ ${ }^{1}$ Aerospace Technology Institute of CARDC, Mianyang 621000, China \\ ${ }^{2}$ Computational Aerodynamics Research Institute, China Aerodynamics Research and Development Center, \\ Mianyang 621000, China \\ ${ }^{3}$ National Innovation Institute of Defense Technology, Academy of Military, Beijing 100071, China
}

Correspondence should be addressed to Pengyu Guo; pengyu.guo@nudt.edu.cn

Received 24 April 2021; Revised 15 September 2021; Accepted 28 September 2021; Published 22 October 2021

Academic Editor: Jaime Lloret

Copyright (c) 2021 Yong Xu et al. This is an open access article distributed under the Creative Commons Attribution License, which permits unrestricted use, distribution, and reproduction in any medium, provided the original work is properly cited.

Radar sea clutters are echoes reflected from a patch of ocean surface, which may significantly interfere with the signals from targets, and seriously degrade the performance of marine radar remote sensing. Thus, it is vital to eliminate the effects of sea clutter. In this paper, we aim at normalizing sea clutter to a uniform level. Firstly, a detailed analysis about the characteristics and differences of clutter and targets is presented; then, we present a heuristic processing scheme which works by solving the task of sea clutter normalization as a classification problem followed by energy normalization. Multiscale and speed-up strategies are incorporated into the dynamic clustering algorithm to found a robust real-time normalization method. Finally, extensive experiments show state-of-the-art results on challenging sea clutter echoes, which demonstrate the feasibility and robustness of the proposed adaptive clustering normalization method.

\section{Introduction}

Marine radar has played a pivotal role in the field of remote sensing applications, especially for maritime navigation, marine rescue, maritime surveillance, and national safety. However, the radar data may be severely affected by sea clutters, which significantly degrade the performance of the radar system. The sea clutters are echoes reflected from the ocean surface, which highly depend on radar frequency, grazing angle, sea state, polarization, rain, wind velocity, and direction $[1,2]$. Additionally, these parameters strongly couple with each other, and the relationships between them are quite complex [3]. Therefore, it is challenging to model the sea clutters by a uniform function or eliminate them through system design. Besides, sea clutter may severely interfere with the signals from targets and seriously degrade the performance of the target detection, particularly for the targets with low speed and low radar cross-section (RCS) [4]. Thus, it is critical to eliminate the effects of sea clutter.
Sea clutter suppression is one of the most significant tasks in radar signal processing. However, it has been a hard problem all the time. In recent years, researches about sea clutter suppression have been shown to achieve improving performance. An exhaustive review of sea clutter progress is outside the scope of our research, but we refer the interested readers to three comprehensive papers $[1,5]$ and [6]. Related to our work, a brief review of sea clutter modeling, filtering, and prediction approaches is presented in the latter paragraphs.

Statistical sea clutter models were proposed basing on the theories of electromagnetic scattering and empirical measurements and observations [1,7]. Sea clutter usually behaves highly non-Gaussian or even spiky, particularly in the case of heavy sea conditions [8]. Consequently, it is tough to model the sea clutter; although, numerous efforts have been devoted to it. The aim of these studies is to improve the understanding of clutter characteristics, then be applied to better eliminate the effects of sea clutter. These 
models include normalized clutter RCS, amplitude statistics, clutter spikes, spatial correlation, and Doppler spectrum [1]. Amplitude statistic models such as Weibull, log-normal [9], $K$-distribution $[10,11]$, non-Rayleigh $[12,13]$ Gaussian, and compound-Gaussian [14] have attracted lots of attentions to describe the amplitudes of sea clutter and also have been confirmed and applied widely.

Besides, some researchers devoted to designing innovative filters to eliminate the influence of sea clutter. In [5], $\mathrm{Lv}$ et al. compared the sea clutter suppression performances of four algorithms, which were developed on cycle cancellation, and singular value decomposition, wavelet weighted reconstruction and decomposition, respectively. In [15], a particle Kalman filter has been used for target detection using marine radar. [16] presented a time-frequency analysis technique to detect target, [17] utilized wavelet for detection, and [18] applied radial basis function (RBF) neural network to detect small objects. Liu et al. proposed a spatial tracking filter [19] to suppress sea clutter. Self-organizing map and hierarchical agglomerative clustering algorithm [20] have been found effective for marine radar clutter classification. In [20], Chen et al. succeeded in identifying both rain contaminated and low backscatter regions. Radon transform was applied to detect small target in [21], as well as fractal analysis theory was employed in $[22,23]$. Liu et al. developed a generalized multichannel adaptive filter to cancellate sea clutter for passive radar sensors and achieved sufficient clutter suppression and target signal preservation [24]. Besides, they proposed a multichannel normalized least-meansquare algorithm to reduce the residual sea clutter using a passive bistatic radar [25].

Moreover, deep neural network (DNN) has been widely used in the radar signal processing field. To predict the amplitudes of sea clutter, Ma et al. [6] taken the advantage of a long short-term memory (LSTM) neural network to found a sea clutter prediction system, which was composed of a preprocessing module followed by a prediction module and could learn the long-term variation characteristics of sea clutter. These approaches eliminated the effects of sea clutter to some extent and achieved improving performance. Nevertheless, sea clutter suppression is not yet a closed problem.

Different from the abovementioned sea clutter modeling, filtering, and prediction methods, our approach is a heuristic processing scheme and works by solving the task of sea clutter normalization as a classification problem followed by energy normalization. The rest of this paper is organized as follows. First, we analyze the amplitude characteristics of sea clutter and targets. Then, we put forward an adaptive clustering method and an energy normalization scheme. For validation, we then test our method with extensive data at different sea states and present the experiment results. Finally, we revisit the major findings of this paper and present some potential applications.

\section{Data Analysis}

In this paper, the datasets were collected on the East China Sea by a civil navigation radar system. Figure 1 illustrates the intensities of sea clutter echoes with respect to four dif- ferent sea states, where the $x$-axis represents the radial distance, and the $y$-axis indicates the echo amplitude. The left column of Figure 1 illustrates the amplitudes of sea clutter without targets in four different sea states. The right column of Figure 1 illustrates the likely targets in sea clutter, which are indicated by the red curves. Through analysis, we obtain the following intuitive but important characteristics:

(i) Both the intensity and fluctuation of sea clutter gradually decrease with respect to the distance. This law is exploited to promote the adaptive clustering task

(ii) Targets may vary from large ships and islands to small submarine periscopes and floating debris, which results in producing different amplitudes and widths, as shown in the right column of Figure 1. Thus, a multiscale strategy is proposed to address this issue

(iii) The pattern of sea clutter is irregular and timevarying. It is hard to model the sea clutter amplitude by an accurate mathematical model

(iv) The sea clutter amplitude is spatially correlated in the high sea states, which may degrade the performance of the known cell-averaging constant false alarm rate (CA CFAR) detector algorithm, as the cell under test is no longer statistically independent of the surrounding cells

Besides, the data in Figure 1 shows that sometimes the amplitude data close to the radar is invalid and sometimes is large enough to saturate the receiver, especially in high sea states. To simplify this problem, in subsequent sections, we substitute the frontal 100 samples by the mean of the samples between positions 100 and 150 . Since one interval corresponds to $2.4 \mathrm{~m}$, the minimum detection distance for our system is about 240 meters. Illuminated by the above characteristics, we consider the sea clutter normalization as a classification process and strive to normalize the sea clutter in a machine learning manner, regardless of the radar parameters and marine environmental parameters.

\section{Methodology}

Clustering is an unsupervised classification method that classifies different categories. Motivated by statistical machine learning theories, we develop a multiscale clustering method to adaptively predict whether there is a likely target in the corresponding bins and then eliminate the effects of sea clutter by normalizing the energy of the data.

Analyzing the sea clutter normalization problem from the perspective of energy normalization, the background means and variances, respectively, describe the potential energy and fluctuation energy of sea clutter. To make an analogy, the energy of sea clutter corresponds to the energy of an object with nonzero altitude accompanied by vibration, and then the potential energy and fluctuation energy correspond to the gravitational potential energy and vibration 

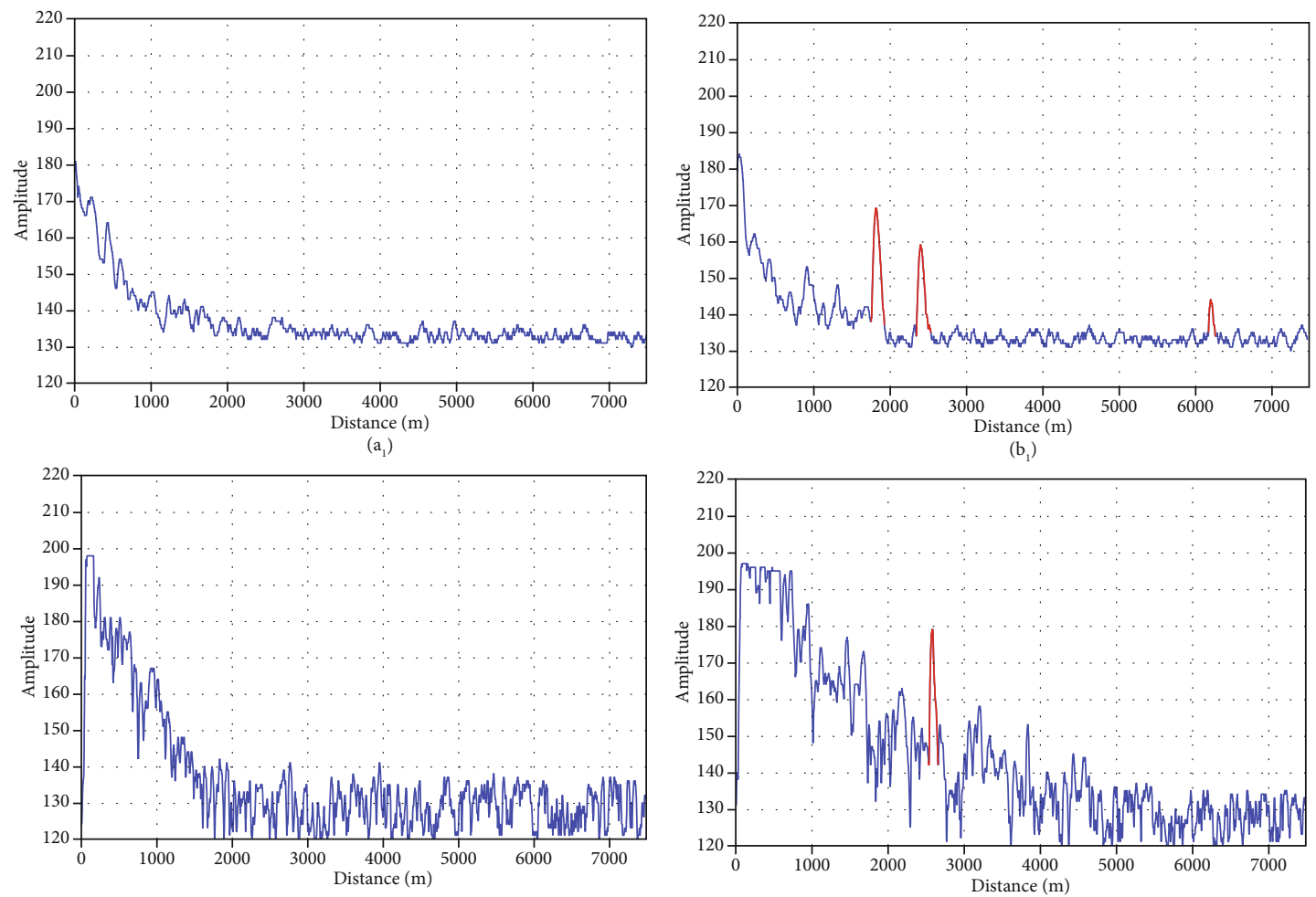

$\left(\mathrm{a}_{2}\right)$
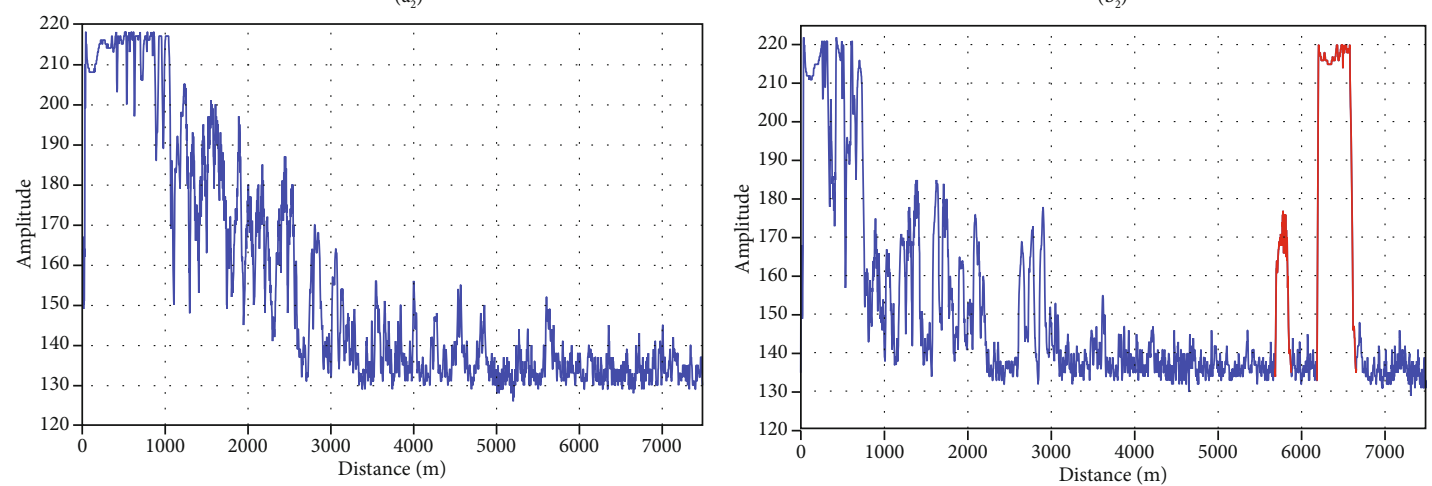

$\left(a_{3}\right)$

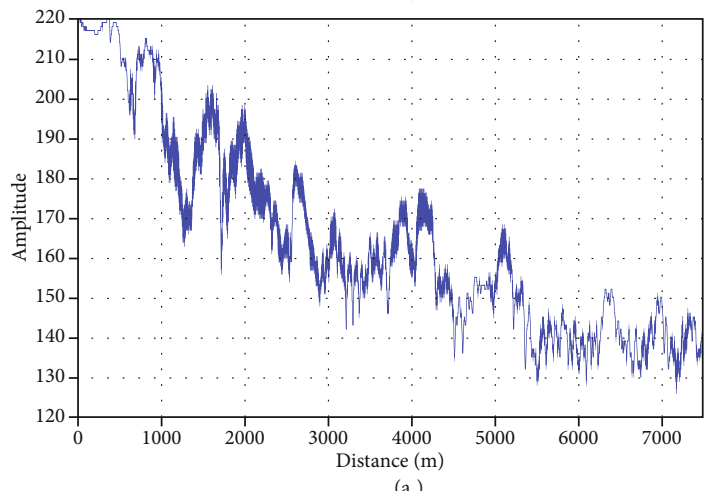

$\left(\mathrm{b}_{3}\right)$

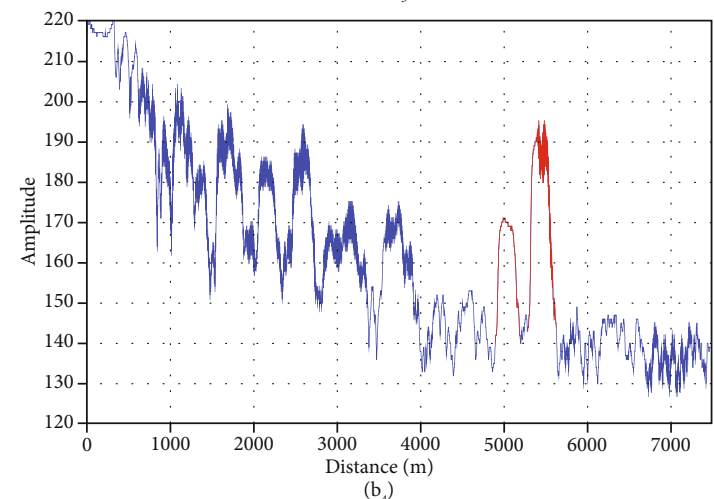

Figure 1: Amplitudes of the marine radar echoes with and without targets. $\left(a_{1}\right),\left(a_{2}\right),\left(a_{3}\right)$, and $\left(a_{4}\right)$ are sea clutter amplitude data without targets recorded in four different sea states $\left(L_{1}, L_{2}, L_{3}, L_{4}\right)$, respectively, and the corresponding sea states satisfy $L_{1}<L_{2}<L_{3}<L_{4}$. $\left(\mathrm{b}_{1}\right),\left(\mathrm{b}_{2}\right)$, $\left(b_{3}\right)$, and $\left(b_{4}\right)$ are amplitudes of the radar data with different targets in the four sea states as well. red curves indicate the likely targets.

energy, respectively. The primary purpose of our research is to normalize the radar sea clutter to a uniform level: for arbitrary distance, the mean of sea clutter amplitudes equals zero, and the corresponding variance equals unit one. Figure 2 illustrates the pipeline of the proposed adaptive clustering-based normalization (ACN) algorithm. 


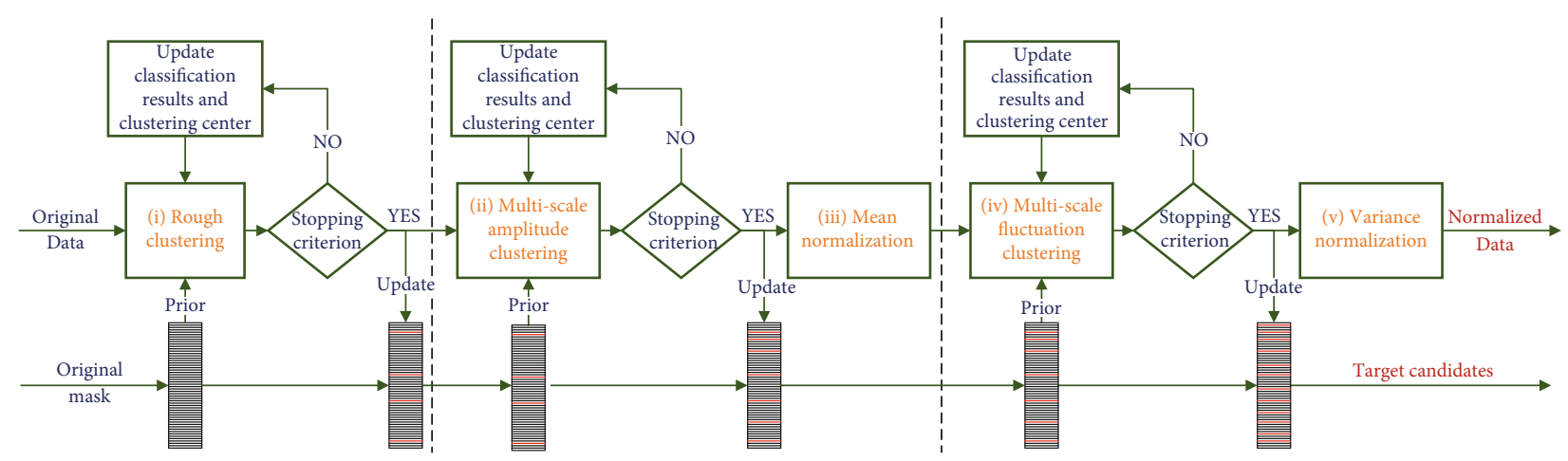

FIgURE 2: Detailed block diagram of ACN framework.

Inputs: one-dimensional sea clutter amplitude data $A$ in a certain direction.

For each bin $b_{i}$ do

Initialize the clustering centers by $C_{0}^{i}=\min \left(\operatorname{mean}\left(b_{i}\right), C_{0}^{i-1}\right)$ and $C_{1}^{i}=\eta \times C_{0}^{i}$;

Iterations $\tau=0, \tau_{\max } ; \eta$ denotes proportional factor;

While $\operatorname{sum}\left(c_{i}^{\tau}\right) \neq \operatorname{sum}\left(c_{i}^{\tau-1}\right)$ and $\tau \leq \tau_{\max }$ do

For each sample $b_{i j}$ compute Manhattan distance with clustering center

$D_{i j}^{y}=\left|b_{i j}-C_{y}\right|$;

If $\widehat{y}_{i j}=\min _{y}\left[D_{i j}^{y}\right]$

End for

Then $b_{i j} \in \widehat{y}_{i j}$ and update classification results $c_{i j}=\widehat{y}_{i j}$;

Update clustering centers respectively:

$C_{1}^{i}=\operatorname{sum}\left(c_{i} \odot b_{i}\right) /\left[\operatorname{sum}\left(c_{i}\right)+1\right], C_{0}^{i}=\operatorname{sum}\left[\left(1-c_{i}\right) \odot b_{i}\right] /\left[m_{i}-\operatorname{sum}\left(c_{i}\right)+1\right]$

+1 for divide protection, $\odot$ explicitly denotes the element-wise multiplication; $\tau=\tau+1$

End while

End for

Integrate classification results $c_{i}$ and clustering centers $C_{0}^{i}, C_{1}^{i}$ in $C, C_{0}$, and $C_{1}$, respectively.

Outputs: classification results $C$ and clustering centers $C_{0}, C_{1}$.

Algorithm 1: Clustering algorithm.

As shown in Figure 2, the proposed adaptive clusteringbased sea clutter normalization scheme consists of four blocks: (i) rough clustering roughly tells apart the targets from sea clutter in a fast manner, the resulting mask denotes by $C$, and the corresponding element $C_{d} \in\{0,1\}$ denotes the class label at distance $d$, where 0 stands for clutter and 1 stands for the suspected targets; (ii) multiscale amplitude clustering takes $C$ as the prior and dynamically classifies the targets and clutter using Algorithm 1 and then estimate the mean value at each distance $d$, denoted by $M_{d}^{*}$; (iii) mean normalization subtracts the effect of background mean by $E_{d}=A_{d}-M_{d}^{*}$, where $A_{d}$ denotes the sea clutter amplitude at distance $d$, and $E_{d}$ denotes the data normalized by mean; (iv) multiscale fluctuation clustering takes the classification results in (ii) as the prior, employs Algorithm 1 to separate targets from sea clutter again, and computes the corresponding variance at each distance $d$, denoted by $V_{d}^{*}$; (v) variance normalization eliminates the effect of background variance by $\widehat{E}_{d}=E_{d} / V_{d}^{*}$, where $\widehat{E}_{d}$ denotes the data further normalized by variance. We maintain the mask $C$ through the whole normalization process, which helps to accelerate the clustering and enhance the robustness of the proposed algo- rithm. Meanwhile, the resulting mask $C$ also indicates the possible targets, which can be taken as the prior for maritime target detection algorithms; although, it may contain false alarm and false dismissal.

3.1. Dynamic Clustering. In this section, we dynamically estimate two statistical characteristics of sea clutter echoes, the background means, and variances. For the sake of time-consuming, first, we employ a rough clustering to roughly exclude the targets from sea clutter. Then, we take the classification results as the prior and utilize a fine dynamic clustering to estimate the statistical ones at each distance. Algorithm 1 describes the pseudocode of the basic clustering algorithm performed in both rough and fine clustering.

The rough clustering process is devoted to roughly telling apart the targets from sea clutter in a fast manner. For this purpose, we divide the one-dimensional amplitude data $A$ into several bins $b_{i}(i=1,2, \cdots, n)$ and compute the integral data of $A$. Each bin consists of $m_{i}$ samples, and $b_{i j}(j=1,2$, $\left.\cdots, m_{i}\right)$ denotes the amplitude of the sample. $y \in\{0,1\}$ denotes the class label, which depicts a binary classification problem, 0 stands for clutter, and 1 stands for likely target. 


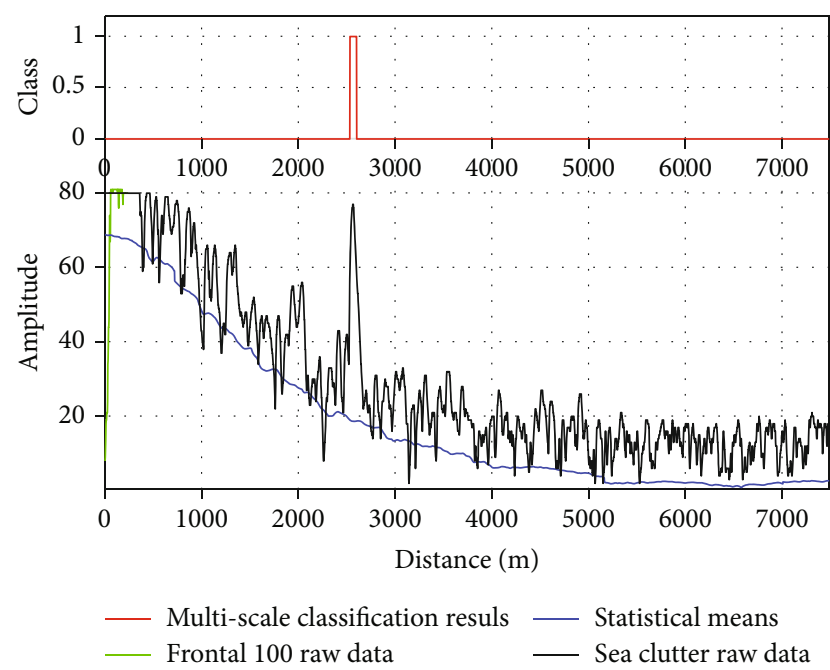

(a)

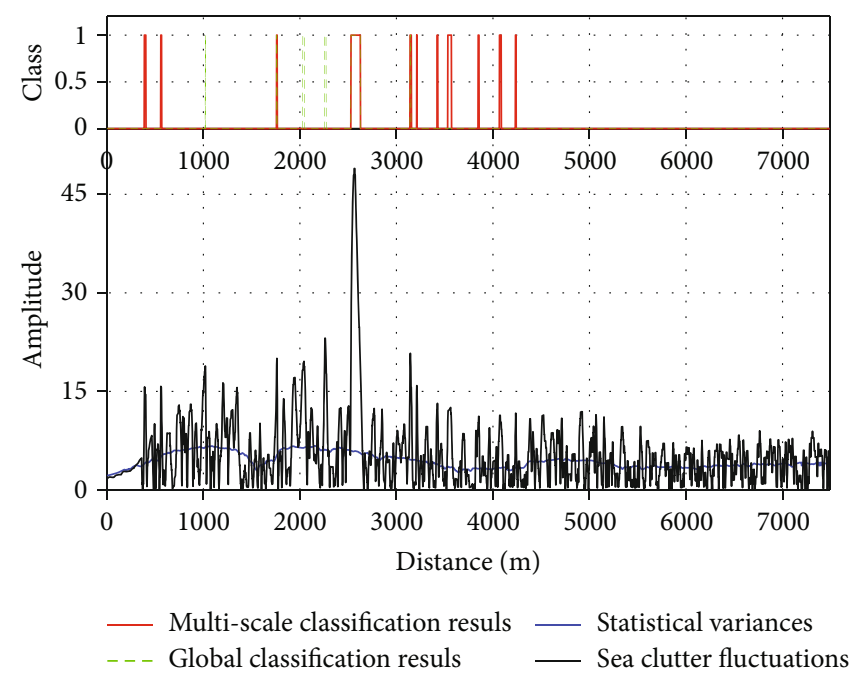

(c)

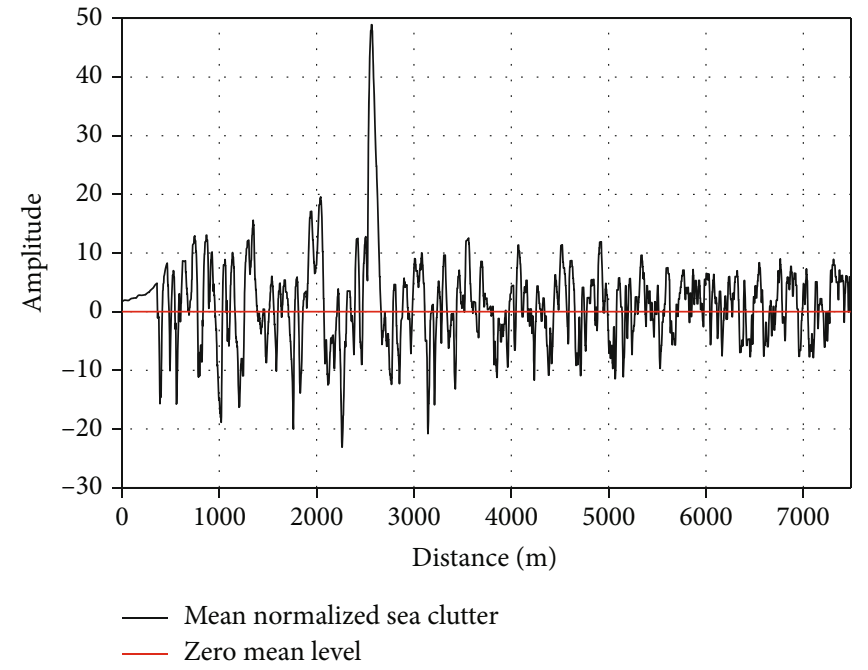

(b)

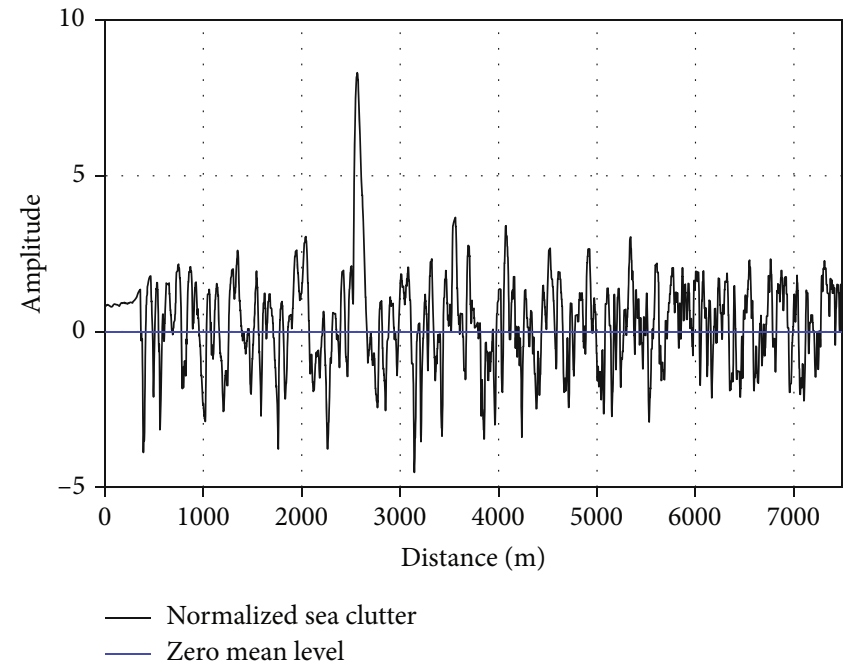

(d)

Figure 3: Clustering and normalization results of the sea clutter amplitude data. (a) The black curve exhibits the amplitude values of the data, the red curve shows the classification results, the blue curve stands for mean values of sea clutter, and the green curve displays the frontal 100 raw data. (b) shows the result of mean normalization. (c) and (d) are the fluctuation clustering and variance normalization results, respectively. The green dotted curve displayed in (c) represents the global clustering results.

$C_{0}$ and $C_{1}$ denote the clustering centers, respectively. $c_{i}$ denotes the corresponding classification results of $b_{i}$, which is produced by Algorithm 1 and the corresponding element $c_{i j} \in\{0,1\}$. Finally, all the rough clustering results are integrated in the mask $C$. Besides, the original mask of rough clustering indicates the prior, and all the elements are set to 0 if there are no priors.

The fine dynamic clustering process aims at excluding the targets from sea clutter in a precise manner. In this module, we cluster at each distance $d$ with the clustering width $w$. To speed up the clustering process, we compute the maskbased integral data of the sea clutter amplitude and take the rough clustering results as a prior. Besides, since both the intensity and fluctuation of sea clutter data gradually decrease with respect to the distance, we exploit this law to dynamically supervise the selection of clustering centers by Equation (1):

$$
C_{0, d+1}^{*}=\min \left(\rho \times C_{0, d}^{*}, C_{0, d+1}\right)
$$

where $C_{0, d+1}$ denotes the clustering center of sea clutter at distance $d+1, \rho$ denotes a proportional factor, and $C_{0, d+1}^{*}$ and $C_{0, d}^{*}$ denote the supervised clustering centers of sea clutter at distance $d+1$ and $d$, respectively. The basic clustering algorithm in this module is the same as Algorithm 1, except for the supervision of clustering centers by Equation (1).

Clustering analysis consists of feature selection, similarity measurement, and clustering cost function. For rough clustering and multiscale amplitude clustering, we choose 


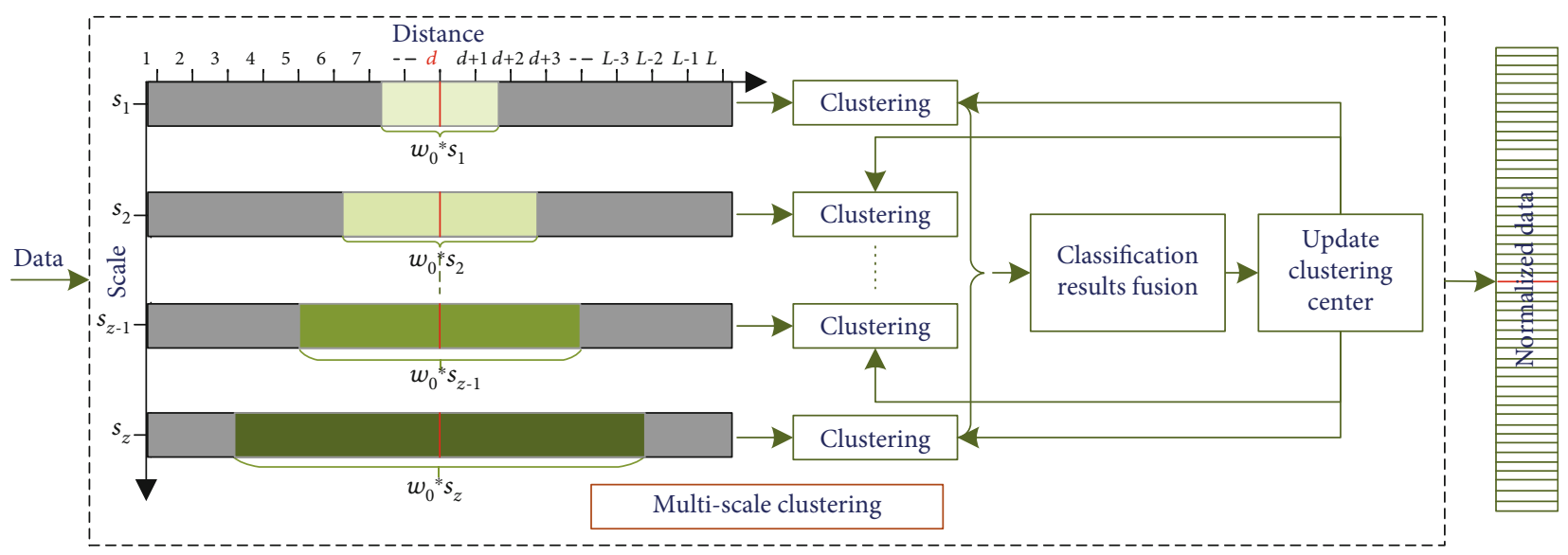

FIGURE 4: Schematic block diagram of the multiscale clustering.

intensity $b_{i j}$ as the clustering feature, and the similarity measurement is formulated by Manhattan distance as Equation (2).

$$
D\left(b_{i j_{1}}, b_{i j_{2}}\right)=\left|b_{i j_{1}}-b_{i j_{2}}\right|,
$$

The clustering cost function is formulated by within class distance, denoted by Equation (3). Then, the clustering results are found by minimizing the cost.

$$
J_{w}=\sum_{j=1}^{m_{i}} c_{i j}\left|b_{i j}-C_{0}\right|+\left(1-c_{i j}\right)\left|b_{i j}-C_{1}\right| \Rightarrow \min .
$$

Algorithm 1 does not explicitly minimize clustering cost $J_{w}$, but the procedure of classification and updating trend to minimize it.

As shown in Figure 2, we successively utilize the results of amplitude clustering and fluctuation clustering to normalize the sea clutter, and the two processes are almost the same. In the fluctuation clustering process, we choose the absolute of the results produced by mean normalization as clustering feature. The statistical mean and variance at discrete distance $d$ can be directly obtained based on the corresponding clustering results by Equation (4):

$$
M_{d}^{*}=C_{0, d}^{m}, V_{d}^{*}=C_{0, d}^{v} .
$$

Here, $M_{d}^{*}$ is the desired background mean, $V_{d}^{*}$ denotes the approximate background variance, and $C_{0, d}^{m}$ and $C_{0, d}^{v}$ denote the clustering centers of the sea clutter in amplitude and fluctuation clustering, respectively. Figure 3 shows the clustering and normalization results of sea clutter amplitude data in a specific direction. Figure 3(b) illustrates the results produced by mean normalization according to $E_{d}=A_{d}-M_{d}^{*}$ . Figure 3(c) indicates the fluctuation bias $\left|E_{d}\right|$. Figure 3(d) shows the final results $\widehat{E}_{d}$, which is computed by $\widehat{E}_{d}=E_{d} /$ $V_{d}^{*}$. The results illustrate that the proposed dynamic clustering scheme can separate the echoes with visual higher local amplitude from the background and accurately estimate the means and variances of sea clutter.
3.2. Adaptive Multiscale Strategy. The widths of target echoes vary from one to hundreds. Thus, if we simply cluster and estimate the characteristics of the background at one fixed width, the results will not satisfy the normalization requirement or even worse. In this section, we propose an adaptive multiscale clustering strategy to deal with different sizes of targets. Figure 4 shows the schematic diagram of the multiscale clustering. For each distance, the corresponding background widths of sea clutter denoted by $s \times w_{0}$, where $w_{0}$ represents the basic width, and $s=\left[s_{1}, s_{2}, \cdots, s_{z}\right]$ represents the scale factors. To integrate multiscale strategy with the dynamic clustering algorithm, we do cluster at each scale using the same clustering centers updated by Equation (5):

$$
C_{0}=\min \left(C_{0}^{s}\right), C_{1}=\operatorname{mean}\left(C_{1}^{s}\right) \text {. }
$$

Here, $C_{0}^{s}$ and $C_{1}^{s}$ denote the clustering centers of background and targets at scale $s$, respectively. Similarly, the multiscale statistical mean and variance at distance $d$ can be obtained by Equation (4). The values are then exploited to normalize the amplitude data at this distance, as shown by the red line in Figure 4 .

\section{Experiments}

4.1. Radar System and Parameters. To validate our approach, we have performed extensive experiments to analyze the normalization performance in various sea states. The testing data are recorded by a two-dimensional noncoherent civil navigation radar system on the East China Sea. The experimental radar system operates at the X-band and works in circular scanning mode, azimuth range $\left[0,360^{\circ}\right]$. The scanning cycle is 2.5 seconds, during which this system emits about 3300 pulses. Therefore, the azimuth resolution is approximately $0.11^{\circ}$. The sampling frequency of our recording instrument is $62.5 \mathrm{MHz}$, which results in range resolution $2.4 \mathrm{~m}$. The distance range we discussed in this paper is set to $r \in[0,7488 m]$, corresponding to 3120 samples.

The approach proposed in this paper is implemented in MATLAB. We perform the experiments on a laptop with Intel(R) Core (TM) $2.60 \mathrm{GHz}$ CPU and $12 \mathrm{~GB}$ RAM. The size of the bins depends on target size and distance 

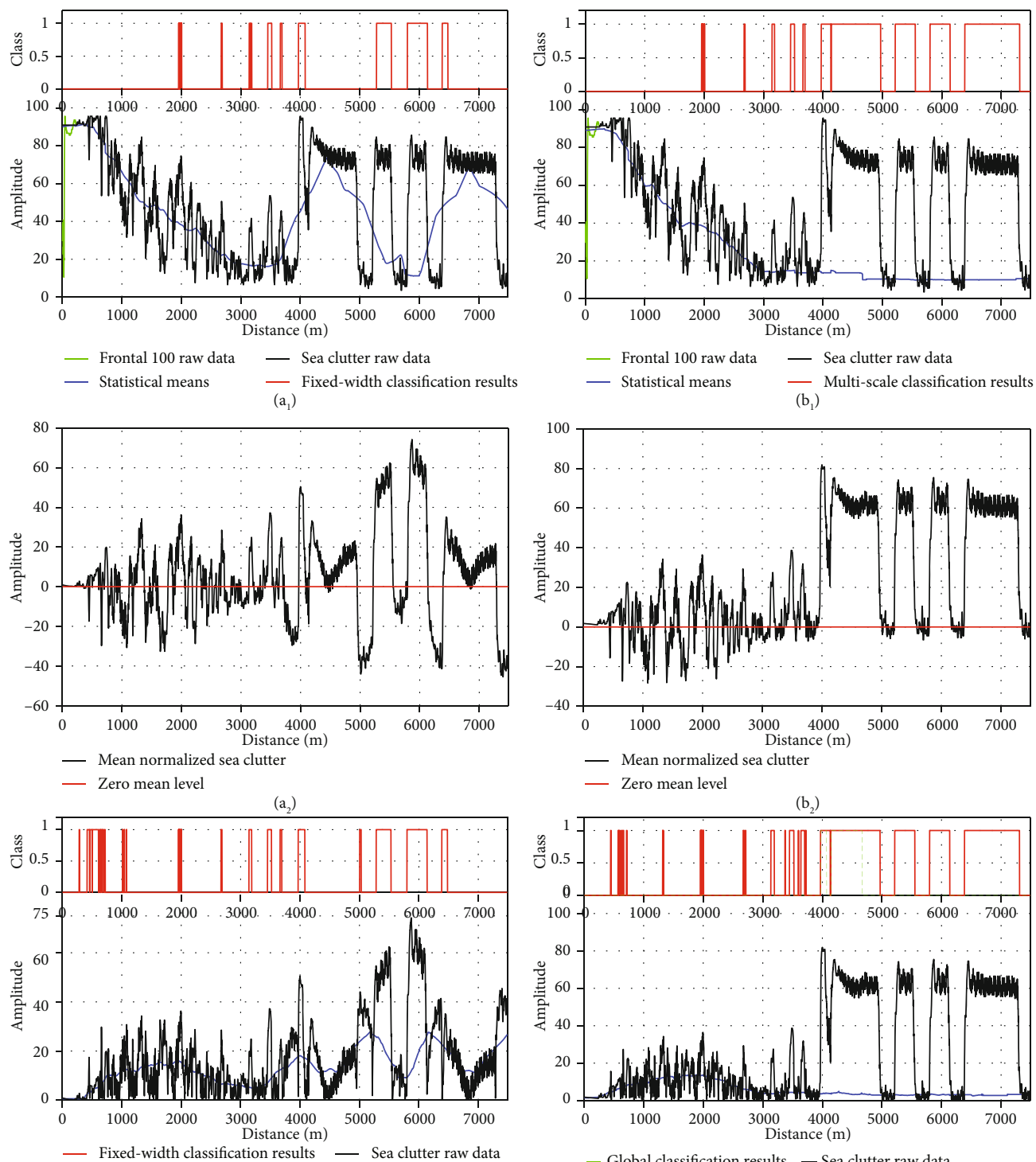

— Zero mean level

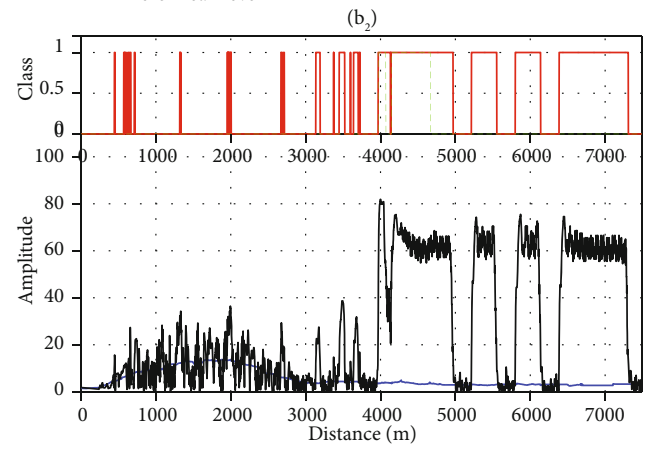

— Statistical mean

$\left(a_{3}\right)$

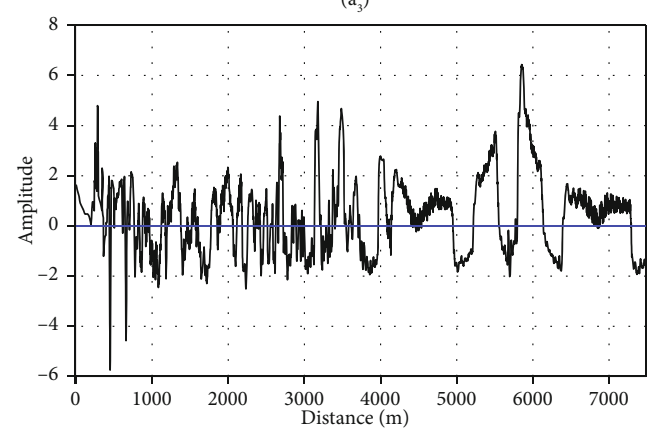

- Global classification results - Sea clutter raw data

- Statistical means _ Multi-scale classification results $\left(b_{3}\right)$

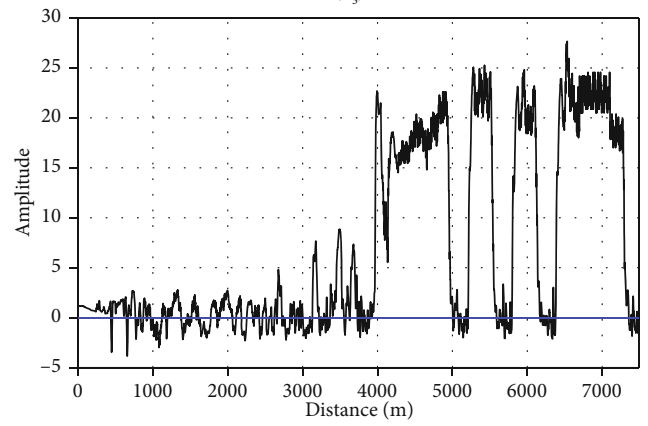

- Normalized sea clutter

- Normalized sea clutter

- Zero mean level

$\left(a_{4}\right)$

$\left(b_{4}\right)$

Figure 5: Comparison of fixed width clustering and multiscale clustering. The left column $(\mathrm{a} *)$ and the right column $(\mathrm{b} *)$ show the results of fixed width clustering and multiscale clustering, respectively. $\left(a_{1}\right)$ and $\left(b_{1}\right)$ indicate the intensity clustering. $\left(a_{2}\right)$ and $\left(b_{2}\right)$ show the mean normalized sea clutter. $\left(a_{3}\right)$ and $\left(b_{3}\right)$ indicate the fluctuation clustering. $\left(a_{4}\right)$ and $\left(b_{4}\right)$ shows the normalized sea clutter. 


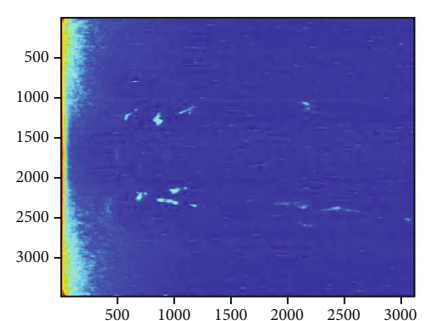

$\begin{array}{llllll}500 & 1000 & 1500 & 2000 & 2500 & 3000\end{array}$
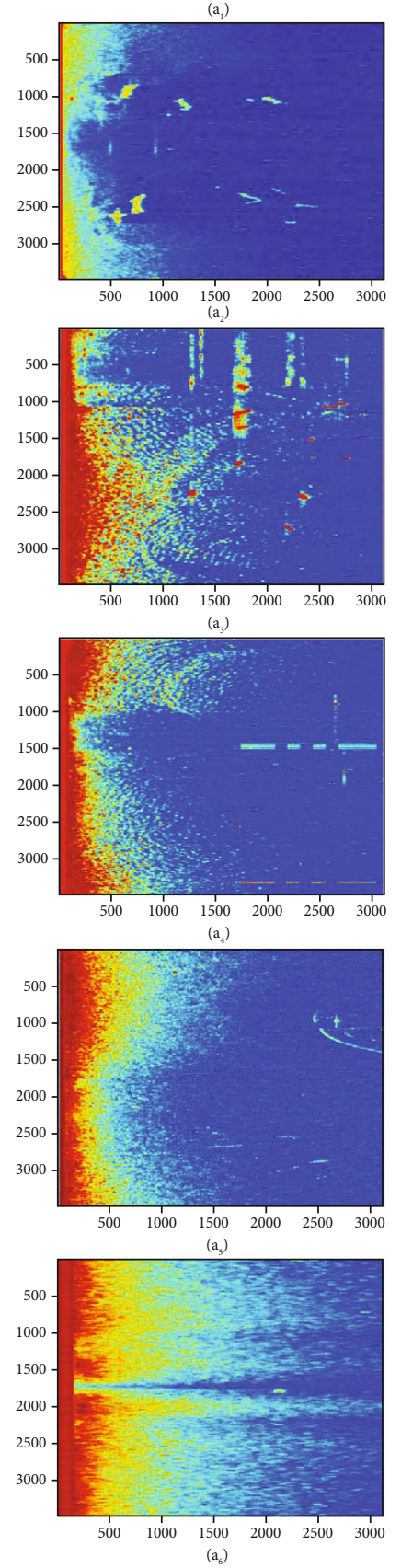
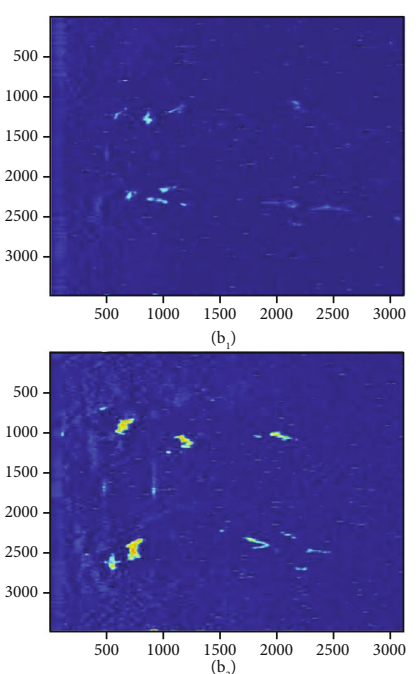

$\begin{array}{llllll}500 & 1000 & 1500 & 2000 & 2500 & 3000\end{array}$
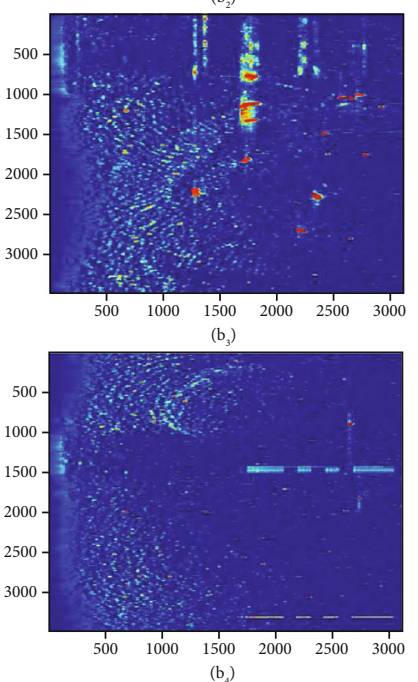

$(\mathrm{b})$
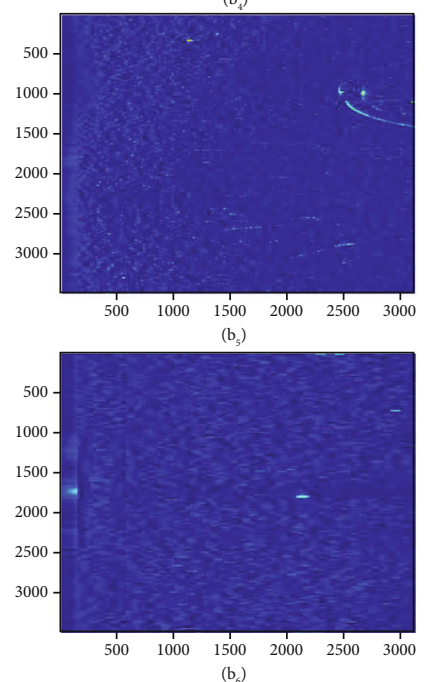
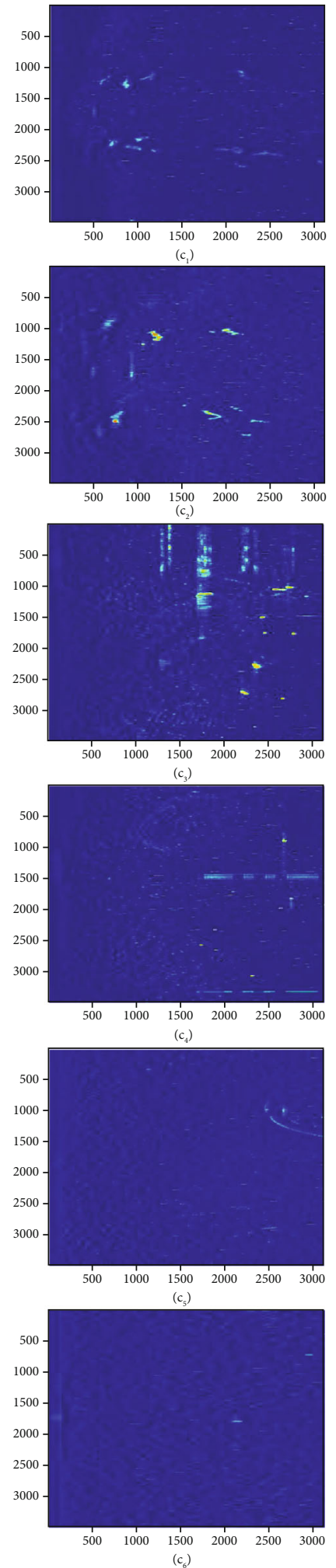

FIGURE 6: Experimental results in six sea states $\left(L_{1}, L_{2}, L_{3}, L_{4}, L_{5}, L_{6}\right)$ within a scanning cycle. The corresponding sea states satisfy $L_{1}<$ $L_{2}<L_{3} \approx L_{4}<L_{5}<L_{6}$. Raw amplitude data, mean normalized data, and mean variance normalized data are shown by (a*), (b*), and (c*), respectively. 

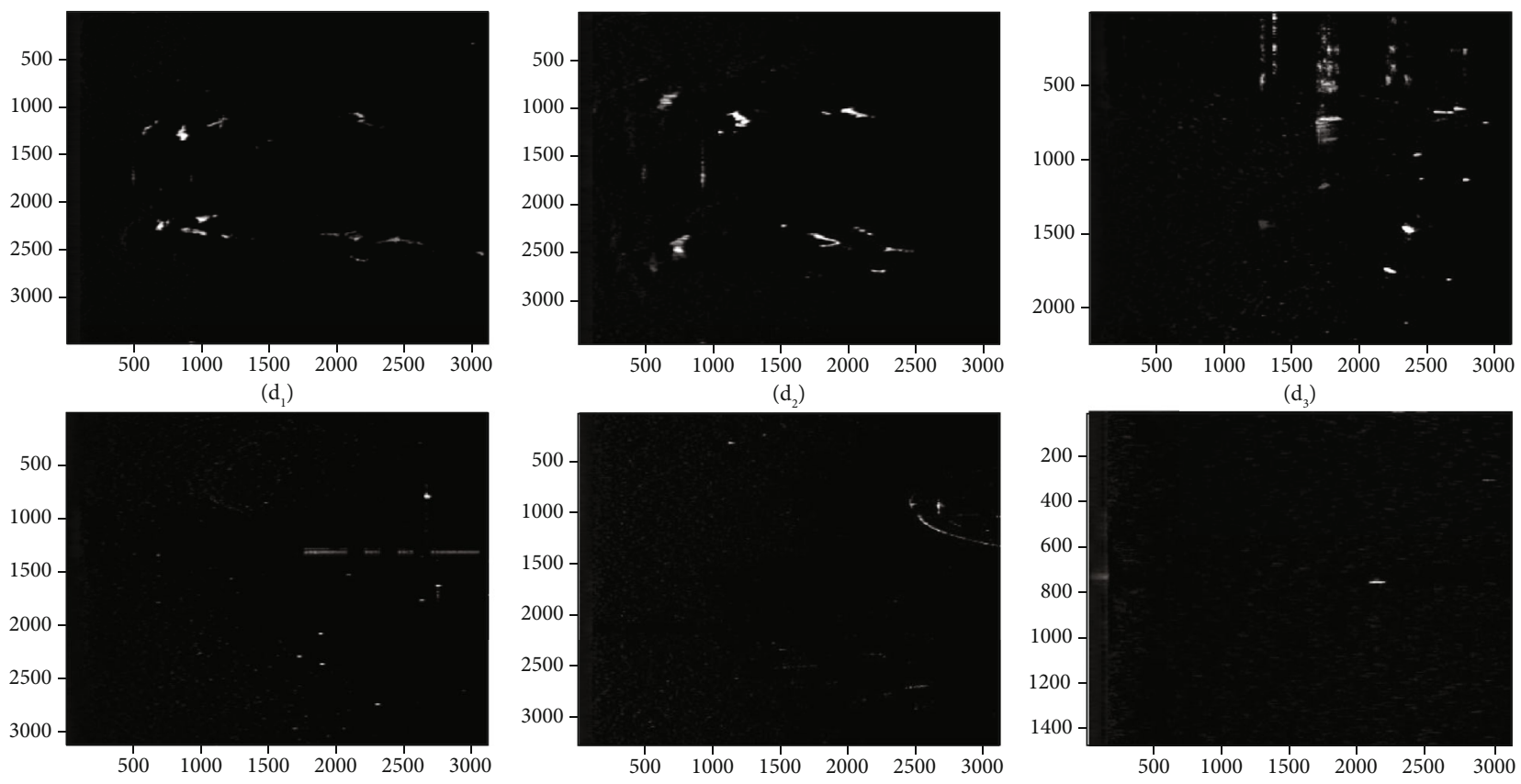

$\left(\mathrm{d}_{4}\right)$

$\left(d_{5}\right)$

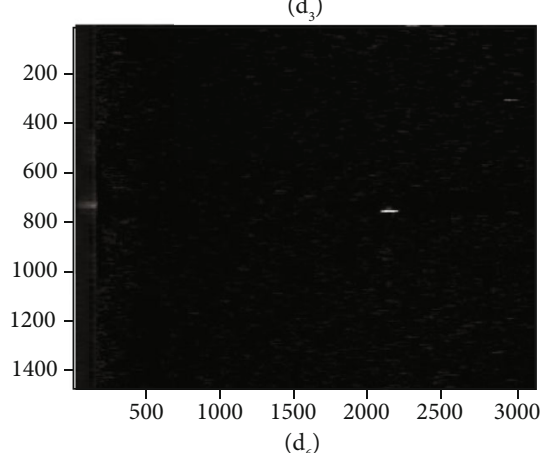

FIGURE 7: Morphological filtering results of the normalized amplitude data shown in Figure 6.

resolution. For rough clustering, the width of the bin is set to $m_{i}=200$ except for the last bin to adapt to different targets sizes; then, the amplitude data at a certain azimuth are divide into 16 bins and $m_{16}=120$. The proportional factor presented in Algorithm 1 is set to $\eta=1.5$ for rough clustering, $\eta=1.3$ for multiscale intensity clustering. These two proportional factors are set to build weak classifiers, which are obtained from extensive experiments based on the echo intensities. According to the 3 -sigma principle of the industry, we set $\eta=3$ for multiscale fluctuation clustering. Besides, the maximum iteration of dynamic clustering is initialized by $\tau_{\max }=5$. For fine dynamic clustering, the proportional factor in equation (1) is set to $\rho=1.1$, and the basic width $w_{0}=150$ and the multiscale factors are set to $s=[1,1.5,2]$; yet, an adhoc scale strategy is needed for the head and tail of sea clutter amplitude data. Additionally, a global clustering is performed before the fine multiscale fluctuation clustering. The iteration of global clustering equals one with clustering centers $C_{0}=\operatorname{mean}(E)$ and $C_{1}=3 \times C_{0}$, and $E$ denotes the fluctuate bias in a certain azimuth. The green dotted lines shown in Figure 3(c) and Figure 5(b $\left.b_{3}\right)$ illustrate the global classification results. These parameters are tested on extensive experiments.

To speed up the clustering process, we compute the mask-based integral data of the sea clutter amplitude. The idea of the integral data is precomputing. The advantage of integral data is that the complexity is constant when calculating the mean of any bin. The complexity of computing integral data increases linearly with the length of the data. Besides, the computational complexity of the proposed method increases linearly with the number of the clustering and the maximum iteration of dynamic clustering. Let $N$ denotes the length of the data, $n$ denotes the number of the clustering, and $\tau_{\max }$ denotes the maximum iteration of dynamic clustering. Then, the computational complexity of the proposed normalization algorithm is approximately $O\left(\left(\tau_{\max } \times n+1\right) \times N\right)$.

4.2. Experimental Evaluation. The first set of experiments aims to explain the pipeline of the presented ACN framework and meanwhile evaluates the performance of the multiscale strategy and clustering centers supervising strategy. As illustrated in Figures 3 and 5 , these tests provide intuitive insights into the operation of adaptive clustering and energy normalization. Figure $5(\mathrm{a} *)$ illustrates the results of fixed width clustering and without utilizing Equation (1) to supervise the clustering center. Figure $5(\mathrm{~b} *)$ show the results produced by the ACN. As shown in the left column of Figure 5, clustering at a fixed width cannot tackle the target with large size, even interfere the desired targets, which results in reducing the signal-to-noise ratio of the targets. However, the results produced by multiscale clustering accurately describe the background mean and variance, and meanwhile, mitigates the effects of sea clutter. Thus, the results demonstrate that the proposed ACN can robustly estimate the sea clutter means and variances and adapts to different sea states and targets.

The second set of experiments exhibit the ensemble performance from a whole scanning cycle before and after normalization. We integrate the amplitude data of one scanning cycle into a two-dimensional image, of which the horizontal axis indicates distance and the vertical axis represents orientation. Figure 6 shows the performance comparison between raw amplitude data, mean normalized data, and mean variance normalized data. As shown in Figure 6, we recorded the data at six different marine areas, and the sea states of 

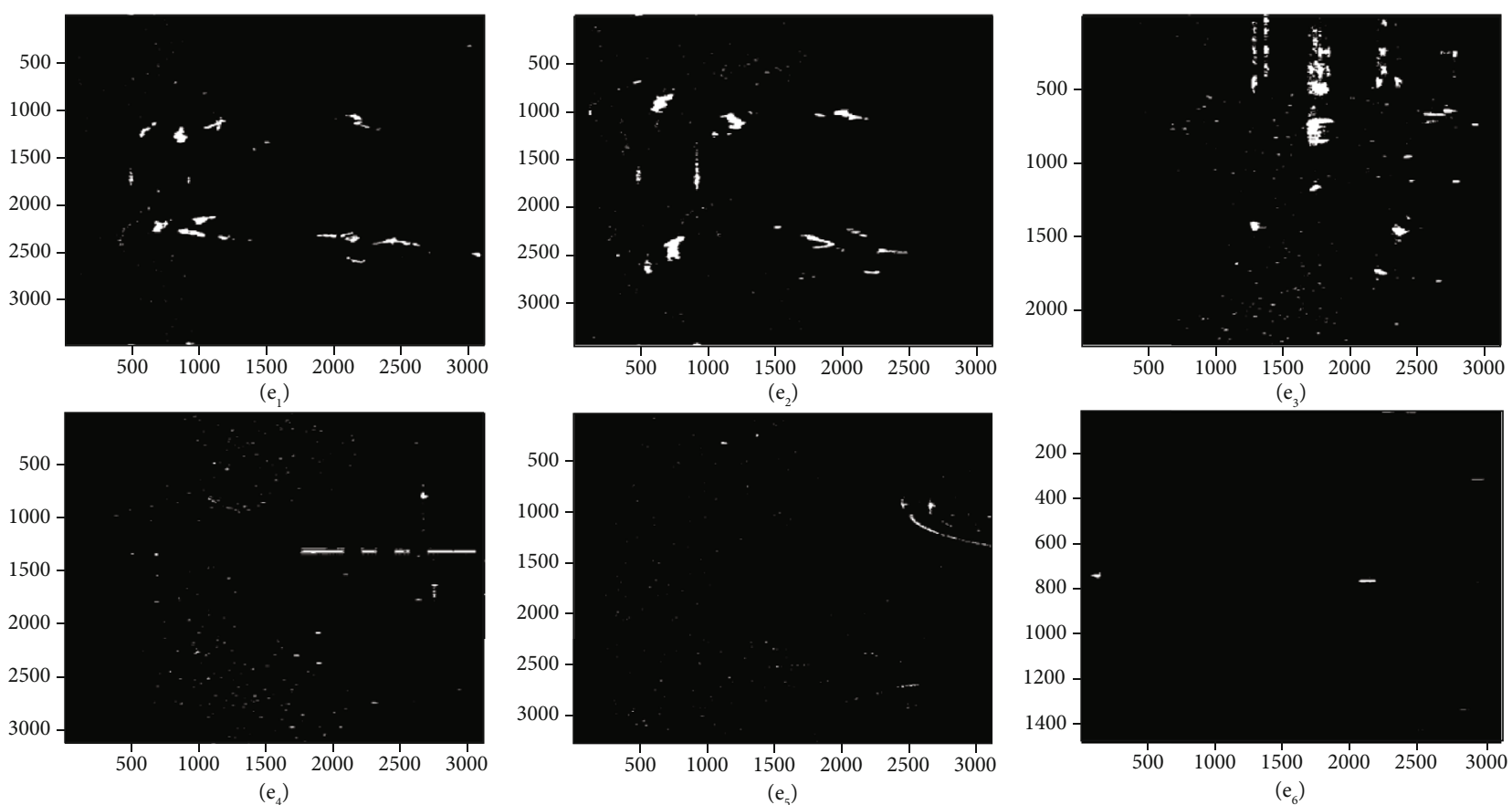

FIgURE 8: Segmentation results of the morphological filtered amplitude data shown in Figure 7, by threshold 3.

the upper rows are lower than that of the bottom column. Intuitively, the left column of Figure 6 indicates that there is a huge patch of strong scattering points, mixed by targets and sea clutters, and the echo intensity is very strong nearby the radar system. However, the middle column of Figure 6 shows that mean normalization has mitigated most of the strong sea clutter. One step further, the right column of Figure 6 exhibits that the effects of fluctuation has been eliminated by variance normalization, with only a few weak points left.

Furthermore, a target detector can be employed to detect targets from the normalized amplitude data. First, we limit the minimum intensity of the normalized data to 0 by setting the negative data to 0 . Besides, to eliminate the influence of the scattering points on target detection, we utilize a morphological filter on the normalized data. Figure 7 shows the corresponding results by corrosion morphological filtering, and the kernel of the morphological filter is a circle with radius of 3 . Since radar sea clutters are normalized to a $u n i$ form level: for arbitrary distance, the mean of sea clutter amplitudes equals zero, and the corresponding variance equals unit one, then the normalized sea clutters can be approximately modeled as a standard normal distribution. According to the 3-sigma principle of the industry, we directly segment the eroded amplitude data shown in Figure 7 , by a threshold 3 , and results are shown in Figure 8. As shown in Figure 8, a simple segmentation can effectively detect the likely targets; though, the segmentation results contain false alarms and false dismissal. More scans are needed to finally confirm the targets and thoroughly eliminate the sea clutter, especially for the scattering points and spiky clutters. Besides, sea clutters severely interfere with the signals from targets, which weaken the relative intensities of target signals, particularly for the targets with low RCS. Therefore, an energy accumulation method should be applied to detect small targets in the target detection task.

\section{Conclusions}

The primary purpose of our research is to eliminate the effects of sea clutter. In this paper, we first analyze the characteristics of sea clutter and targets. Then, we propose an intuitive, simple, but high-quality normalization method, which exploits a dynamic clustering algorithm to classify targets and sea clutter in terms of echo amplitude and fluctuation, then estimates the statistical means and variances of sea clutter by utilizing the classification results, and finally, employs the estimated means and variances to normalize sea clutter to a uniform level. In addition, we integrate multiscale and speed-up strategies with the dynamic clustering algorithm to form a robust adaptive clustering approach in handling different sizes of targets. Two sets of experiments show state-of-the-art results on challenging sea clutter echoes, which demonstrate the effectiveness and robustness of the proposed ACN algorithm.

The high quality and efficiency of our method make it suitable for real-time object detection and tracking applications. Thus, the proposed ACN can be considered as a preprocessing technique for these radar remote sensing applications. Besides, similar data have also been captured in many different fields. Hence, the method proposed in this paper could be employed to normalize signals in those fields.

\section{Data Availability}

The marine radar data used to support the findings of this study have been deposited in the GitHub repository 
https://github.com/mbsdtf/marine-radar-data-sets/releases/ tag/v1.0.

\section{Conflicts of Interest}

The authors declare that there is no conflict of interest regarding the publication of this paper.

\section{Acknowledgments}

The authors would like to thank the editors and anonymous reviewers for their valuable comments and suggestions on the paper. This research was partly supported by the Science and Technology Innovation 2030 Major Project under grant no. 2020AAA0104801 and the Natural Science Foundation of China under grant no. 61903364.

\section{References}

[1] K. D. Ward, R. J. A. Tough, and P. W. Shepherd, "Modelling sea clutter: correlation, resolution and non-Gaussian statistics," in Radar Systems (RADAR 97), pp. 95-99, Edinburgh, UK, 1997.

[2] X. Chen, W. Huang, C. Zhao, and Y. Tian, "Rain detection from X-band marine radar images: a support vector machine-based approach," IEEE Transactions on Geoscience and Remote Sensing, vol. 58, no. 3, pp. 2115-2123, 2020.

[3] S. Haykin, R. Bakker, and B. W. Currie, "Uncovering nonlinear dynamics-the case study of sea clutter," Proceedings of the IEEE, vol. 90, no. 5, pp. 860-881, 2002.

[4] R. F. Gasparovic and J. R. Jensen, "Sea Clutter Statistics and Detection Performance of High-Altitude Maritime Search Radars," 2004, SRO-04-01, JHU-APL.

[5] M. Lv and C. Zhou, "Study on sea clutter suppression methods based on a realistic radar dataset," Remote Sensing, vol. 11, no. 23 , p. $2721,2019$.

[6] L. Ma, J. Wu, J. Zhang et al., "Sea clutter amplitude prediction using a long short-term memory neural network," Remote Sensing, vol. 11, no. 23, p. 2826, 2019.

[7] M. S. Greco and S. Watts, "Radar Clutter Modeling and Analysis," Academic Press Library in Signal Processing: Volume 2 Communications and Radar Signal Processing, vol. 2, pp. 513-594, 2014.

[8] T. P. Leonard, I. Antipov, and K. D. Ward, "A comparison of radar sea clutter models," in 2002 International Radar Conference, Edinburgh, UK, 2003.

[9] S. Watts, "Radar sea clutter: recent progress and future challenges," in International Conference on Radar, IEEE, pp. 1016, Adelaide, SA, Australia, 2008.

[10] S. Sayama and M. Sekine, "Log-normal, log-Weibull and Kdistributed sea clutter," IEICE Transactions on Communications, vol. 85, pp. 1375-1381, 2002.

[11] K. D. Ward, R. J. A. Tough, and S. Watts, Sea Clutter: Scattering, the K Distribution and Radar Performance, Institution of Engineering and Technology, 2006.

[12] E. Conte, M. Longo, and M. Lops, "Modelling and simulation of non-Rayleigh radar clutter," IEE Proceedings F Radar and Signal Processing, vol. 138, no. 2, pp. 121-130, 1991.

[13] E. Jakeman and P. Pusey, "A model for non-Rayleigh Sea Echo," IEEE Transactions on Antennas and Propagation, vol. 24, no. 6, pp. 806-814, 1976.
[14] F. Gini, "Performance analysis of two structured covariance matrix estimators in compound-Gaussian clutter," Signal Processing, vol. 80, no. 2, pp. 365-371, 2000.

[15] S. Chen and W. Huang, "Maneuvering target tracking from nautical radar images using particle-Kalman filters," Journal of Electromagnetic Waves and Applications, vol. 27, no. 18, pp. 2366-2378, 2013.

[16] T. Thayaparan and S. Kennedy, "Detection of a manoeuvring air target in sea-clutter using joint time-frequency analysis techniques," IEE Proceedings-Radar, Sonar and Navigation, vol. 151, pp. 19-30, 2004.

[17] G. Davidson and H. D. Griffiths, "Wavelet detection scheme for small targets in sea clutter," Electronics Letters, vol. 38, no. 19, pp. 1128-1130, 2002.

[18] H. Leung, N. Dubash, and N. Xie, "Detection of small objects in clutter using a GA-RBF neural network," IEEE Transactions on Aerospace and Electronic Systems, vol. 38, pp. 98-118, 2002.

[19] J. Y. Liu, H. D. Meng, H. Zhang, and X. Wang, "Radar sea clutter suppression and target indication with a spatial tracking filter," Tsinghua Science and Technology, vol. 15, no. 2, pp. 228234, 2010.

[20] X. Chen and W. Huang, "Identification of rain and lowbackscatter regions in X-band marine radar images: an unsupervised approach," IEEE Transactions on Geoscience and Remote Sensing, vol. 58, no. 6, pp. 4225-4236, 2020.

[21] J. Carretero-Moya, J. Gismero-Menoyo, A. Asensio-Lopez, and A. Blanco-del-Campo, "Small-target detection in sea clutter based on the radon transform," in 2008 International Conference on Radar, pp. 610-615, Adelaide, SA, Australia, 2008.

[22] J. Hu, J. Gao, F. L. Posner, Y. Zheng, and W. W. Tung, "Target detection within sea clutter: a comparative study by fractal scaling analyses," Fractals, vol. 14, no. 3, pp. 187-204, 2006.

[23] K. Sun, Analysis of Sea Clutter Fractal Characteristic and Application Research of Target Detection, Nanjing University of Science \& Technology, 2013.

[24] S. Liu, Y. Ma, and Y. Huang, "Sea clutter cancellation for passive radar sensor exploiting multi-channel adaptive filters," IEEE Sensors Journal, vol. 19, no. 3, pp. 982-995, 2019.

[25] T. Shan, Y. Ma, R. Tao, and S. Liu, "Multi-channel NLMSbased sea clutter cancellation in passive bistatic radar," IEICE Electronics Express, vol. 11, no. 20, article 20140872, 2014. 